\section{Propagation of Radio Waves from Cosmical Sources}

WHEN a radio wave passes through an un-ionized medium its ray path is rectilinear. On passing through such ionized layers, which usually surround the Earth, its trajectory is satisfactorily determined by Snell's law :

$$
\mu \rho \sin i=\rho \|=\sin i_{0}=\text { constant }
$$

which is valid for any point of the ray path. For the sake of simplicity the radius of curvature $\rho_{m}$ of the maximum of electron density is taken as the unit of length. The significance of the parameters used follows from Fig. 1.

According to ( 1 ) and Fig. 1, the rectilinear part of the ray path must be tangential to the sphere defined by $\rho_{\|}$, everywhere in the un-ionized medium. Thus a ray which pesses through spherical ionized layers of the same centre of curvature $O$ is turned about this centre of curvature by the angle $R$ (Fig. 1).

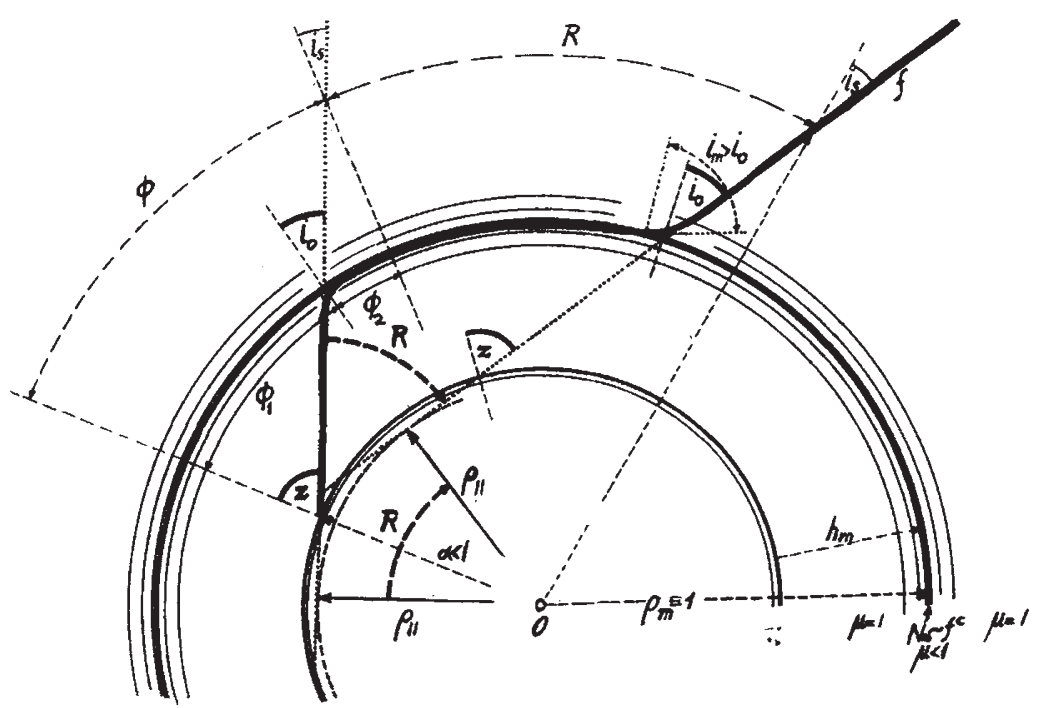

Fig. 1. Parameters used in formulø 1 and $2 . f$ is the wave frequency, $f$ the critical frequency of the layer.

$$
\mu=\sqrt{1-\left(\frac{f c}{f}\right)^{2}} \text { and } \rho=\frac{6,400 \mathrm{~km} .+h}{6,400 \mathrm{~km} .+h_{m}}, h \text { and } h_{m} \text { being in } \mathrm{km} \text {. }
$$

Fig. 2. The true and the equivalent layer, introduced in order to avoid discrepancies that could appear as the electron density is ususily expressed in cm. ${ }^{3}$ while the introduced unit of length is pm. In this equin in order to form a lazer of uniform electron dengity $(N$. The thick into formula 2

trons present in the layer, but not upon the irregularity in electron distribution with height, which up to the present time has made the calculation of refraction uncertain. Formula 2 is valid even for sharp gradients of electron density, and for parabolic types of layer, $\sigma=\frac{4}{3} \Delta$, it goes over into Bailey's formula ${ }^{3}$.

The theoretical result, mentioned above, is in good agreement with experimental results obtained from the signal records of the first artificial satellite. The angular distance of the satellite $\phi+R$ was determined from formulæ 1 and 2 and Fig. 1 as follows :

$$
\begin{gathered}
a \sin z=\left(a+h_{m}\right) \sin i_{0} \\
\sin i_{\text {sat. }}=\frac{a}{a+h_{\text {sat. }}} \sin z \\
\varphi_{1}=z-i_{0}, \quad \varphi_{2}=i_{0}-i_{\text {sat. }}, \\
\varphi=\varphi_{1}+\varphi_{2}=z-i_{\text {sat }} . \\
\text { and } \sin i_{0} \leqslant \mu_{m} \rho_{m}
\end{gathered}
$$

For this angle of refraction $R$ the following simple formula has been derived ${ }^{1}$ :

$$
R=\frac{90^{\circ}}{\pi}\left(\frac{f c}{f}\right)^{2} \frac{\sin i_{0}}{\cos ^{3} i_{0}} \cdot \sigma
$$

where again $p_{m}=1, i_{0}$ is the angle of incidence of the rectilinear parts of the ray path, related, however, to this level $\rho_{m}=1$, and the equivalent thickness $\sigma$ of the layer is to be determined according to Fig. 2.

Formula 2 is valid for $\frac{f c}{f \cos i_{0}}<0.4$ and $\Delta<p_{m} / 5$.

For $f c / f \cos i_{0}>0.4$ the true refraction is greater than the calculated one, and it increases to infinity; this case, dealing with a perpetual propagation of radio waves around the Earth along one of the two different levels of the ionosphere, has been treated previously ${ }^{2}$.

The refraction formula 2 has the advantage that it depends only upon the total content of all free elec- corresponds to the minimum of the up-curve. In accordance with the calculated values the received signal disappeared usually sooner on $20 \mathrm{Mc}$./s. than on $40 \mathrm{Mc} / \mathrm{s}$., and only in cases when the signal was corming from countries where for $20 \mathrm{Mc} . / \mathrm{s} . f \doteq f^{c}$ was long-distance propagation of the signal around the Earth from the magnetic equator observed.

Summarizing, the rectilinear part of a ray path becomes rotated about the centre of curvature of the ionized layer through which it has penetrated by the angle of refraction $R$. This deviation $R$ depends only upon $i_{0}, f \circ / f$ and the content of free electrons preaent in the whole layer, but it is independent of their distribution.

\section{E. Chvojkoví}

Astronomical Institute of Czechoslovakia, Ondř̈ejov. Nov. 9.

${ }^{1}$ Chrojková, E., Bull. Astron. Czech., [9, 1 (1958)].

${ }^{2}$ Chvojková, E., Bull. Astron. Czech., 5, 104, 110 (1954).

${ }^{8}$ Bailey, D. K., J. Mag. Atm. Elect., 53, 41 (1948). 